\title{
Visita domiciliar como suporte da enfermagem na diálise peritoneal: revisão integrativa
}

Home visit as a support for nursing in peritoneal dialysis: an integrative review Visita domiciliaria como asistencia de enfermería en la diálisis peritoneal: revisión integradora

\author{
Laudilene Cristina Rebello Marinho' id https://orcid.org/0000-0001-6585-6481 \\ Fabiana Tomé Ramos ${ }^{1}$ id https://orcid.org/0000-0002-2770-2630 \\ Rogério Carvalho de Oliveira ${ }^{1}$ id https://orcid.org/0000-0003-1270-7372 \\ Jaqueline Teixeira Caramoni ${ }^{1}$ io https://orcid.org/0000-0002-1359-3058 \\ Cassiana Mendes Bertoncello Fontes ${ }^{1}$ id https://orcid.org/0000-0002-6579-8637
}

Como citar: JT, Fons F, Olveira RC, Caramoni JT, Fontes CM. Visita domiciliar como suporte da enfermagem na diálise peritoneal: revisão integrativa. Acta Paul Enferm. 2020;33:APE20190139.

DOI http://dx.doi.org/10.37689/actaape/2020A001395

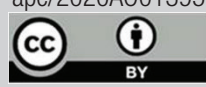

Descritores

Diálise peritoneal; Visita domiciliar; Educação em saúde; Tratamento domiciliar; Enfermagem domiciliar

Keywords Peritoneal dialysis: House calls: Health education; Residential treatment; Home health nursing

Descriptores Diálisis peritoneal; Visita domiciliaria; Educación en salud; Tratamiento domiciliário; Cuidados de enfermería en el hogar

Submetido 21 de Maio de 2019

Aceito

28 de Janeiro de 2020

\section{Autor correspondente}

Fabiana Tomé Ramos

E-mail: fabiana-tr@hotmail.com

\section{Resumo}

Objetivo: Identificar na literatura como a visita domiciliar é utilizada pelo enfermeiro como ferramenta do cuidado em dialise peritoneal domiciliar.

Métodos: Revisão integrativa da literatura de artigos em periódicos, a partir de uma questão norteadora, no período de janeiro de 2014 a janeiro de 2019. A busca bibliográfica foi realizada nas bases Scielo; Web of Science, Pubmed, Scopus, Embase e Cinahl.

Resultados: Identificaram-se 10 artigos de acordo com as etapas da revisão e as respostas à pergunta norteadora. A visita domiciliar realizada pelo enfermeiro foi descrita como ferramenta de adesão ao tratamento. A prevenção de complicações, como a peritonite, foi citada pelos autores, que em sua maioria, eram médicos e enfermeiros.

Conclusão: 0 enfermeiro assiste integralmente ao paciente e família e, assegura o tratamento proposto com fidedignidade. As intervenções de enfermagem no domicílio previnem eventos adversos e frequente avaliação de indicadores do tratamento. Estimular estudos primários nessa área é imprescindível, visto que poucos estudos sobre a temática foram identificados na presente revisão.

\section{Abstract}

Objective: To identify in the literature how home visit is used by nurses as a tool of care in home peritoneal dialysis.

Methods: An integrative review of the literature of articles in journals, from a guiding question, from January 2014 to January 2019. The bibliographic search was carried out at Scielo, Web of Science, Pubmed, Scopus, Embase, and CINAHL databases.

Results: Ten articles were identified according to the review steps and the answers to the guiding question. The home visit performed by nurses was described as a treatment adherence tool. Complication prevention, such as peritonitis, was cited by the authors, who were mostly physicians and nurses.

Conclusion: Nurses assist patients and family members in full and ensure the proposed treatment with reliability. Nursing interventions at home prevent adverse events and frequent assessment of treatment indicators. Boosting primary studies in this area is essential, since few studies on the subject have been identified in the present review.

\section{Resumen}

Objetivo: Identificar en la literatura cómo se utiliza la visita domiciliaria del enfermero como herramienta de cuidado en diálisis peritoneal domiciliaria. 
Métodos: Revisión integradora de la literatura de artículos en revistas especializadas, a partir de una pregunta orientadora, en el período de enero de 2014 a enero de 2019. La búsqueda bibliográfica fue realizada en las bases Scielo, Web of Science, Pubmed, Scopus, Embase y Cinahl.

Resultados: Se identificaron diez artículos de acuerdo con las etapas de la revisión y las respuestas a la pregunta orientadora. La visita domiciliaria realizada por el enfermero se describió como herramienta de adherencia al tratamiento. La prevención de complicaciones, como la peritonitis, fue citada por los autores que, en su mayoría eran médicos y enfermeros.

Conclusión: El enfermero asiste al paciente y a la familia de forma integral y garantiza que el tratamiento propuesto sea fidedigno. Las intervenciones de enfermería en el domicilio previenen eventos adversos y una evaluación frecuente de indicadores del tratamiento. Es imprescindible estimular estudios primarios en esta área, ya que se identificaron pocos estudios sobre esta temática en la presente revisión.

\section{Introdução}

Segundo dados do sistema Único de Saúde (SUS), em 2015, o número estimado de usuários em tratamento dialítico que estão em "manutenção e acompanhamento domiciliar submetidos à diálise peritoneal automatizada (DPA) e diálise peritoneal ambulatorial contínua (DPAC)" estava próximo a 6.126 usuários no Brasil. ${ }^{(1)} \mathrm{A}$ realização do programa educacional em DPA e DPAC depende da intervenção de um enfermeiro habilitado para a condução do procedimento que será realizado em domicílio. ${ }^{(1-3)}$

Há anos a enfermagem ensina e aconselha pacientes em vários contextos do cuidado à saúde a serem desenvolvidos no domićlio. Devido à base de conhecimentos, atuaçáo na equipe de saúde e proximidade com o paciente, o enfermeiro é um dos profissionais de saúde mais preparado para prover educação em saúde. ${ }^{(1)}$

A visita domiciliar (VD) é um método de trabalho em enfermagem que tem como objetivo principal levar ao indivíduo, em seu domicílio, uma assistência e orientação sobre sua saúde. Os resultados são atingidos por meio de: educação em saúde na supervisão de cuidados prestados pela família, ou por um de seus membros como cuidador; prestação de cuidados de enfermagem; identificaçâo de dados familiares sobre as condiçóes de saneamento da moradia, por meio de entrevistas e observaçóes; orientação sobre a prestaçáo dos cuidados no domicílio e assuntos de higiene geral. ${ }^{(3)}$

Durante a VD, o enfermeiro deve estar atento à busca da adequaçáo do ambiente para a realização da dialise peritoneal (DP), mas é necessário que, ao considerar as condiçôes domiciliares, não se perca o objeto da sua atençấo: o ser humano que demanda cuidado.

Sáo escassos os estudos que trazem a temática da DP como indicaçáo primária de tratamento, quando comparado a hemodiálise (HD). A peritonite é um dos fatores que é responsável por essa situação, devido a falha no tratamento da DP. Isso pode ser revertido com a realizaçáo de treinamento e capacitação da equipe de enfermagem para o manejo da técnica de DP domiciliar, visitas periódicas e supervisão podem evitar esses problemas. ${ }^{(4-6)}$

Dessa maneira surgiu o questionamento da revisão: "Como se descreve a VD realizada pelo enfermeiro à pacientes em DP quanto a adesão ao método dialítico, a prevenção de complicaçôes e a sua eficácia como ferramenta de apoio?"

O objetivo da revisão foi identificar na literatura como a visita domiciliar é utilizada pelo enfermeiro como ferramenta do cuidado em dialise peritoneal domiciliar.

\section{Métodos}

As etapas da revisão integrativa foram: definição da pergunta norteadora da revisão; busca sistematizada de artigos científicos nas bases de dados nacionais e internacionais; análise na íntegra, identificação e categorização das evidências nos artigos selecionados; interpretação dos resultados e apresentação da revisão ou síntese do conhecimento. ${ }^{(7)}$

A questão norteadora foi: "Como se descreve a VD realizada pelo enfermeiro à pacientes em $\mathrm{DP}$ quanto a adesão ao método dialítico, a prevençâo de complicaçôes e a sua eficácia como ferramenta de apoio?”.

O levantamento bibliográfico foi realizado pelos autores sob a orientaçáo da bibliotecária de referência na biblioteca da universidade e o período de busca foi delimitado de janeiro de 2014 a janeiro de 2019. A busca foi realizada nas bases de dados Scielo; Web of Science, Pubmed, Scopus, Embase e Cinahl. Os termos utilizados para a estratégia de busca foram selecionados no Descritores em 
Ciências da Saúde (DeCS) e no Medical Subject Headings (MeSH) em combinação com os operadores booleanos AND e OR, seguindo-se as recomendações do Preferred Reporting Items for Systematic reviews and Meta-Analyses - (PRISMA).

(8) Foram elaboradas equaçóes para a buscas em português e inglês na Scielo e nas bases Pubmed, Web of Science, Scopus, Embase e Cinahl.

A estratégia em inglês, espanhol e português direcionada à plataforma Scielo foi: (Peritoneal Dialysis OR Diálisis Peritoneal OR Diálise Peritoneal) AND (Home Visit OR Visita Domiciliaria OR Visita Domiciliar).

A estratégia em inglês foi elaborada para Pubmed, Web of Science, Scopus, Embase e Cinahl foi: (Dialyses, Peritoneal OR Dialysis, Peritoneal OR Peritoneal Dialyses) AND (Call, House OR Calls, House OR House Call OR Home Visits OR Home Visit OR Visit, Home OR Visits, Home).

Os critérios de inclusão foram: artigos publicados na íntegra disponíveis eletronicamente gratuitamente; em idiomas português, inglês e ou espanhol; e que respondessem a pergunta norteadora da revisáo.

Os critérios de exclusão foram: cartas ao editor; relatos de caso; editoriais; artigos em duplicidade; aqueles que no título já não se referiam ao tema proposto.

Para análise na íntegra dos artigos incluídos foram utilizados os instrumentos Strengthening the Reporting of Observational Studies in Epidemiology (STROBE) (9) para os estudos quantitativos e Consolidated Criteria for Reporting Qualitative Studies (COREQ) ${ }^{(10)}$ para os qualitativos; a atribuição do nível de evidência foi realizada baseado nos seguintes critérios: nível 1- revisões sistemáticas ou metanálise de relevantes ensaios clínicos; nível 2 - evidências de pelo menos um ensaio clínico randomizado controlado bem delineado; nível3 - ensaios clínicos bem delineados sem randomização; nível 4 - estudos de coorte e de caso-controle bem delineados; nível 5 - revisão sistemática de estudos descritivos e qualitativos; nível 6 - evidências derivadas de um único estudo descritivo ou qualitativo; nível 7 - opinião de autoridades ou comitês de especialistas incluindo interpretaçôes de informaçốes não baseadas em pesquisas. ${ }^{(11)}$

Foram encontrados 235 artigos, após a pré-seleção, seguiu-se a leitura dos títulos e resumos. $\mathrm{O}$ fluxograma (Figura 1) demonstra os 10 artigos incluídos de acordo com as etapas da revisão e às respostas à pergunta.

\section{Resultados}

Os artigos incluídos e analisados na íntegra distribuem-se assim: um na Web of Science, três na

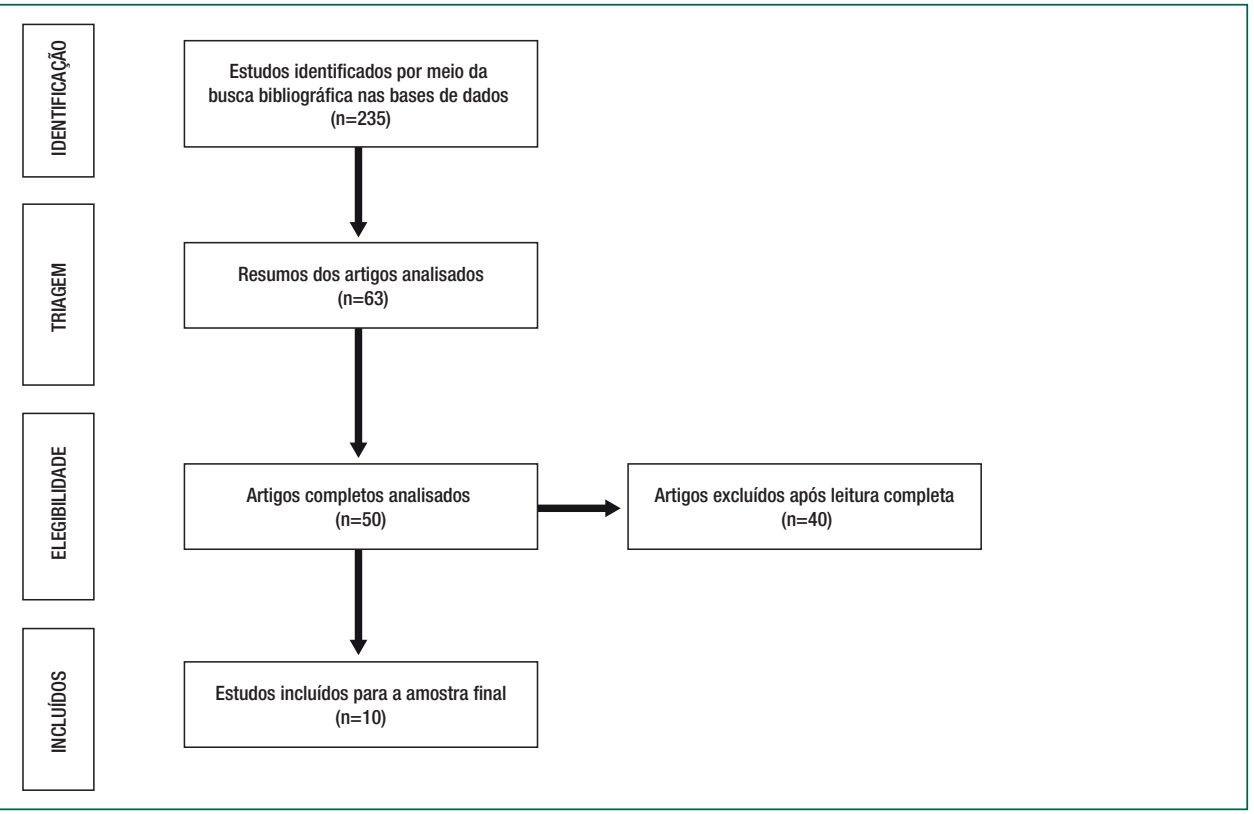

Figura 1. Fluxograma de seleção dos estudos conforme o Preferred Reporting Items for Systematic Reviews and Meta Analyses (PRISMA) 
Quadro 1. Artigos incluídos na revisão integrativa com a estratégia de busca

\begin{tabular}{|c|c|c|c|c|c|c|c|c|}
\hline Ano/ País & Título & Autoria & Tipo de Estudo & $\begin{array}{l}\text { Base de } \\
\text { dados }\end{array}$ & Periódico & $\begin{array}{l}\text { Nivel de } \\
\text { Evidencia }\end{array}$ & STROBE & COREQ \\
\hline $\begin{array}{l}2014 \\
\text { Canadá }\end{array}$ & $\begin{array}{l}\text { Evaluating the benefitsof home-based } \\
\text { Peritoneal dialysis }\end{array}$ & François K, Bargman JM ${ }^{(14)}$ & Revisão narrativa & Scopus & \begin{tabular}{|l|} 
International Journal \\
of Nephrology and \\
Renovascular Disease \\
\end{tabular} & VII & - & - \\
\hline $\begin{array}{l}2014 \\
\text { Chile }\end{array}$ & \begin{tabular}{|l|} 
Perfil biosociodemografico y valoracion \\
de necesidades en pacientes em \\
dialisis peritoneal \\
\end{tabular} & \begin{tabular}{|l|} 
Varela JU,Vidal \\
MR,Pedreros MC'(16)
\end{tabular} & \begin{tabular}{|l} 
Quantitativa, \\
descritiva, de corte \\
transversal
\end{tabular} & Scielo & Ciencia y enfermería & VI & 20 & - \\
\hline $\begin{array}{l}2014 \\
\text { Colômbia }\end{array}$ & $\begin{array}{l}\text { Safety automated peritoneal dialysis- } \\
\text { DPA in children by primary } \\
\text { caregivers home } \\
\end{array}$ & Montilla L et al(17) & Transversal & Scielo & Enfermería Global & VI & 19 & - \\
\hline $\begin{array}{l}2014 \\
\text { Itália }\end{array}$ & \begin{tabular}{|l|} 
Home Visit Program Improves \\
Technique \\
Survival in Peritoneal Dialysis \\
\end{tabular} & Martino, F et al(18) & $\begin{array}{l}\text { Retrospectivo de } \\
\text { caso-controle }\end{array}$ & Scopus & BloodPurif & VI & 21 & - \\
\hline $\begin{array}{l}2014 \\
\text { Brasil }\end{array}$ & $\begin{array}{l}\text { Nursing practice in home care: na } \\
\text { integrative literature review }\end{array}$ & $\begin{array}{l}\text { Andrade AM, Silva KL, } \\
\text { Seixas CT, Braga PP(12) }\end{array}$ & Revisão integrativa & Embase & RevBrasEnferm & VII & - & - \\
\hline $\begin{array}{l}2015 \\
\text { Brasil }\end{array}$ & $\begin{array}{l}\text { As práticas assistências de } \\
\text { enfermagem na diálise peritoneal: } \\
\text { uma revisão }\end{array}$ & $\begin{array}{l}\text { Araújo FEA Neto VLS, } \\
\text { Mendonça AEOM, Lins } \\
\text { TLCE, Granjeiro, FML }{ }^{(13)} \\
\end{array}$ & Revisão integrativa & Scopus & RevEnferm UFPI. & VII & - & - \\
\hline $\begin{array}{l}2015 \\
\text { México }\end{array}$ & \begin{tabular}{|l|} 
Conocimiento teórico y apego al \\
procedimiento de diálisis peritoneal Del \\
paciente o su familiar.
\end{tabular} & Meza WJMG et al ${ }^{(19)}$ & Descritivo transversal & Scielo & EnfermNefrol & VI & 21 & - \\
\hline $\begin{array}{l}2015 \\
\text { Reino Unido }\end{array}$ & $\begin{array}{l}\text { Lack of motivation : A new Modifiable } \\
\text { risk factor for peritonitis in patientes } \\
\text { under do ing peritoneal dialysis. }\end{array}$ & $\begin{array}{l}\text { KlaraPaudel, } \\
\text { GirishNamagondlu, KPG } \\
\text { et al(20) }\end{array}$ & Descritivo & Embase & Journalof Renal Care & VI & 21 & - \\
\hline $\begin{array}{l}2017 \\
\text { Brasil }\end{array}$ & $\begin{array}{l}\text { The home visit in peritoneal dialysis: } \\
\text { relevant aspects to nursing care. }\end{array}$ & Cunha, LP et al(3) & \begin{tabular}{|l|} 
Qualitativa, \\
exploratório- \\
descritiva \\
\end{tabular} & $\begin{array}{l}\text { Web of } \\
\text { Science }\end{array}$ & J. res.: fundam. care. & VI & - & 30 \\
\hline $\begin{array}{l}2017 \\
\text { Canadá }\end{array}$ & $\begin{array}{l}\text { Evaluation of real-time use of } \\
\text { electronic patient-reported outcome } \\
\text { data by nurses with patients in home } \\
\text { dialysis clinics. }\end{array}$ & $\begin{array}{l}\text { Makaroff Kara Schick and } \\
\text { Anita E. Molzahn }{ }^{(15)}\end{array}$ & $\begin{array}{l}\text { Estudo simultâneo, } \\
\text { longitudinal e de } \\
\text { métodos mistos }\end{array}$ & Embase & $\begin{array}{l}\text { Health Services } \\
\text { Research }\end{array}$ & V & 22 & - \\
\hline
\end{tabular}

Scopus, três na Embase e três na Scielo. Em relação ao país de origem, observa-se no quadro 1 uma maior produção do Brasil $(30 \%),{ }^{(3,12,13)}$ seguida pelo Canadá $(20 \%),{ }^{(14,15)}$ ficando os demais países com apenas uma publicação cada. Quanto aos tipos de estudo encontrados, verifica-se que a maioria (60\%) foram de estudos descritivos. Em relação à autoria, os autores que mais publicaram sobre a área temática estão os enfermeiros docentes (70\%) e os médicos nefrologistas (30\%). Referente ao ano de publicação, (50\%) dos artigos foram publicados em 2014, (30\%) no ano de 2015 e (20\%) em 2017. Quanto às bases de dados, a Scielo, Embase e Scopus apresentaram maior número de artigos (n3), seguido pela Web of Science. Foi identificado estudos descritivos, quantitativos, qualitativos e de revisóes de literatura. Segundo o nível de evidencia, nota-se que mais da metade $(60 \%)$ apresentaram nível VI, (30\%) apresentou nível VII e (10\%) nível $\mathrm{V}$. O quadro 1 resume a caracterização e as evidências analisadas nos artigos inclusos: título do artigo; identificação dos autores e ano da publicação; objetivo do estudo; resultados e conclusóes/ recomendaçóes.
Autores concluem que o programa de Visita Domiciliar é uma grande ferramenta para otimização do cuidado, melhorando a sobrevida do paciente com a redução da peritonite e hospitalização. ${ }^{(3,9,18)}$

Um estudo sobre dialise peritoneal domiciliar relata que essa modalidade é efetiva na sobrevida dos pacientes, reduzindo o custo da hemodiálise. As vantagens são o empoderamento e autonomia do paciente na realização dos próprios cuidados, além de requerer a capacitação profissional. ${ }^{(14)}$

Os autores referem sobre a importância de ações educacionais pelo enfermeiro na assistência ao paciente dialítico para a minimização riscos. Existe também a necessidade de formação específica profissional e preparo para a atenção domiciliar. ${ }^{(12,13)}$

Um estudo mostrou que as crianças são vulneráveis a múltiplos fatores de risco, havendo a necessidade de estratégias de avaliação. A equipe interdisciplinar e a de enfermagem em especial, abordaram diretrizes de cuidados para o DPA, minimizando riscos e complicaçóes, e aumentando a segurança do paciente. ${ }^{(17)}$

Um estudo mostra déficits de conhecimento no procedimento de dialise peritoneal, tanto do pa- 
Quadro 2. Síntese dos resultados de acordo com os estudos e respectivos temas a que pertencem

\begin{tabular}{|c|c|c|c|}
\hline Título & Objetivo (s) & Resultados & Conclusões \\
\hline $\begin{array}{l}\text { Evaluating the benefits of } \\
\text { home-based peritoneal } \\
\text { dialysis. }\end{array}$ & $\begin{array}{l}\text { Discutir os benefícios da DP crônica, } \\
\text { realizada pelo paciente ou por um } \\
\text { cuidador em casa }\end{array}$ & $\begin{array}{l}\text { Um sistema de saúde só terá benefício econômico sustentado } \\
\text { pelo aumento do uso da DP se as estratégias de reembolso } \\
\text { iguais para diferentes modalidades de diálise forem apoiadas } \\
\text { pelo reconhecimento médico dos benefícios clínicos e } \\
\text { psicossociais da DP e, pelo treinamento apropriado de } \\
\text { profissionais de saúde para pacientes com conhecimento e } \\
\text { liberdade para realizar diálise em casa. }\end{array}$ & $\begin{array}{l}\text { A maioria dos pacientes com insuficiência renal pode ser } \\
\text { tratada com diálise peritoneal, uma modalidade de diálise } \\
\text { efetiva com sobrevida global similar e com custo menor em } \\
\text { comparação à hemodiálise. Uma vantagem adicional da diálise } \\
\text { peritoneal em casa é o empoderamento intrínseco do paciente. } \\
\text { Todo paciente deve receber educação sobre todas as opções } \\
\text { de diálise. }\end{array}$ \\
\hline $\begin{array}{l}\text { Perfil } \\
\text { biosociodemografico } \\
\text { y valoracion de } \\
\text { necesidades en } \\
\text { pacientes em dialisis } \\
\text { peritoneal }\end{array}$ & $\begin{array}{l}\text { Conhecer as características } \\
\text { biossociodemográficas e a satisfação } \\
\text { das necessidades dos pacientes em } \\
\text { tratamento com diálise peritoneal } \\
\text { do Hospital Las Higueras em } \\
\text { Talcahuano, Chile. }\end{array}$ & $\begin{array}{l}0 \text { grupo foi composto igualmente por homens e mulheres, idade } \\
\text { média de } 48 \text { anos, mais da metade vive sem companheiro e é } \\
\text { chefe de família, a maioria tem filhos e moram com } 4 \text { ou mais } \\
\text { pessoas ( } 57,5 \%) \text {. A maioria completou o ensino médio, } 22,5 \% \\
\text { estão trabalhando e recebem remuneração. Quase todos têm } \\
\text { saneamento básico e vivem na area urbana. As principais } \\
\text { necessidades não satisfeitas correspondem a "eliminar bem" } \\
\text { e "evitar o perigo", as demais estão satisfeitas entre } 57,5 \% \text { e } \\
\text { 92,5\%; Dentro deste intervalo, as necessidades mais afetadas } \\
\text { eram manter a temperatura corporal e recriar. }\end{array}$ & $\begin{array}{l}\text { Os resultados sugerem a implementação e reforço de planos } \\
\text { educacionais mais rigorosos aos já existentes em nosso centro } \\
\text { de saúde, tendendo a evitar danos físicos e psicológicos, além } \\
\text { de manter uma boa eliminação corporal, através do feedback } \\
\text { em cada controle ou visita domiciliar feita ao paciente. }\end{array}$ \\
\hline $\begin{array}{l}\text { Safety automated } \\
\text { peritoneal dialysis-DPA } \\
\text { in children by primary } \\
\text { caregivers home. }\end{array}$ & $\begin{array}{l}\text { Avaliar a segurança das crianças } \\
\text { da DPA em casa, através de } \\
\text { treinamento para cuidadores. }\end{array}$ & $\begin{array}{l}\text { Foram avaliadas } 12 \text { crianças entre } 5-18 \text { anos, } 60 \% \text { com } \\
10-15 \text { anos, em condições ambientais, } 83 \% \text { residiam em } \\
\text { domicílios concluídos, } 17 \% \text { em construção. Em relação ao nível } \\
\text { educacional da cuidador, } 58 \% \text { possuem o ensino fundamental }\end{array}$ & $\begin{array}{l}\text { Crianças com DPA são vulneráveis a múltiplos fatores de } \\
\text { risco, sendo necessárias estratégias para avaliação. A equipe } \\
\text { interdisciplinar abordou as diretrizes de cuidados de enfermagem } \\
\text { para o DPA, tornando-se uma maneira eficaz de minimizar } \\
\text { riscos e complicações, desencadeando o desenvolvimento de } \\
\text { habilidades para orientar a segurança do DPA em casa. }\end{array}$ \\
\hline $\begin{array}{l}\text { Home Visit Program } \\
\text { Improves Technique } \\
\text { Survival in Peritoneal } \\
\text { Dialysis. }\end{array}$ & $\begin{array}{l}\text { Examinar o efeito de um programa } \\
\text { regular de visita domiciliar sobre os } \\
\text { resultados clínicos como sobrevida } \\
\text { da técnica, peritonite e taxas de } \\
\text { hospitalização durante um ano de } \\
\text { seguimento. }\end{array}$ & $\begin{array}{l}0 \text { grupo caso foi significativamente mais velho ( } p=0,048) \text {, com } \\
\text { menor grau de autonomia ( } p=0,033 \text { ) que } 0 \text { grupo controle. } \\
\text { Durante o período de observação, observou-se } 11 \text { episódios de } \\
\text { falha técnica. } \\
\text { Foi encontrado uma redução significativa na taxa de falha da } \\
\text { técnica no grupo caso ( } p=0,004) \text {. } \\
\text { A análise de sobrevida mostrou uma extensão significativa do } \\
\text { tratamento da DP nos pacientes apoiados pelo programa de } \\
\text { visita domiciliar ( } 52 \text { vs. } 48,8 \\
\text { semanas, } p=0,018) \text {. Não houve diferença entre os dois } \\
\text { grupos em termos de peritonite e hospitalização, entretanto, as } \\
\text { tendências em direção a uma redução de } \\
\text { taxas de peritonite, bem como a prevalência e duração dos } \\
\text { relacionados com problemas de DP foram identificados no } \\
\text { grupo caso. }\end{array}$ & $\begin{array}{l}\text { O programa de visita domiciliar } \\
\text { melhora a sobrevida dos pacientes com DP e poderia reduzir a } \\
\text { taxa de peritonite Gram-positiva e hospitalização. }\end{array}$ \\
\hline $\begin{array}{l}\text { Nursing practice in home } \\
\text { care: na integrative } \\
\text { literature review }\end{array}$ & $\begin{array}{l}\text { Analisar a produção científica acerca } \\
\text { da atuação do enfermeiro na atenção } \\
\text { domiciliar em saúde. }\end{array}$ & $\begin{array}{l}\text { Identificou-se que a atuação do enfermeiro na atenção } \\
\text { domiciliar possui complexidade e diversidade de ações com } \\
\text { uso de tecnologias leves, leve-duras especialmente, e duras. } \\
\text { Os desafios relacionados ao processo formativo para a atenção } \\
\text { domiciliar estão relatados na literatura. } 0 \text { enfermeiro utiliza } \\
\text { conhecimento e recomendações científicas aliadas à reflexão } \\
\text { na prática. }\end{array}$ & $\begin{array}{l}\text { A atuação do enfermeiro no espaço domiciliar é fundamental e } \\
\text { ampla. As ações relacionais e educacionais se destacam, sendo } \\
\text { necessárias inclusive nos cuidados técnicos, predominando a } \\
\text { necessidade de formação para a atenção domiciliar. }\end{array}$ \\
\hline $\begin{array}{l}\text { As práticas assistências } \\
\text { de enfermagem na } \\
\text { diálise peritoneal: uma } \\
\text { revisão }\end{array}$ & $\begin{array}{l}\text { Identificar na literatura as práticas } \\
\text { assistenciais de enfermagem ao } \\
\text { paciente em procedimento de diálise } \\
\text { peritoneal. }\end{array}$ & $\begin{array}{l}\text { No processo de diálise peritoneal, os cuidados podem ser } \\
\text { categorizados da seguinte forma: pré, intra e pós-procedimento, } \\
\text { tudo isso conforme a necessidades humanas básicas do } \\
\text { paciente. No âmbito domiciliar o enfermeiro deve avaliar o } \\
\text { ambiente da casa e sugerir as modificações necessárias. }\end{array}$ & $\begin{array}{l}0 \text { enfermeiro exerce um papel indispensável na assistência ao } \\
\text { paciente em tratamento dialítico, com o intuito de minimizar } \\
\text { riscos e melhorar a vida dos pacientes. }\end{array}$ \\
\hline $\begin{array}{l}\text { Conocimiento teórico y } \\
\text { apego al procedimiento } \\
\text { de diálisis peritoneal Del } \\
\text { paciente o su familiar. }\end{array}$ & $\begin{array}{l}\text { Determinar o nível de conhecimentos } \\
\text { teóricos e fixação para o } \\
\text { procedimento de diálise do paciente } \\
\text { ou a família. }\end{array}$ & $\begin{array}{l}0 \text { Grupo Paciente obteve uma taxa mais elevada }(75,35 \%) \text { do } \\
\text { que o Grupo Familiar }(71,45 \%) \text {. } \\
\text { Na técnica em geral houve aderência de } 80,3 \% \text { dos passos, } \\
\text { obtendo o GF maior índice }(81,92 \%) \text { do que GP }(78,63 \%) \text {. }\end{array}$ & $\begin{array}{l}\text { Os pacientes e famílias mostraram déficit em ambas as áreas, } \\
\text { devendo ser avaliadas periodicamente para detectar falhas na } \\
\text { técnica e conhecimento, que predispõem o paciente a uma } \\
\text { complicação evitável. }\end{array}$ \\
\hline $\begin{array}{l}\text { Lack of motivation: } \\
\text { A new Modifiablerisk } \\
\text { factor for peritonitis in } \\
\text { patientes underdoing } \\
\text { peritoneal dialysis }\end{array}$ & $\begin{array}{l}\text { Determinar se o padrão de moradia, } \\
\text { a técnica de troca de DP ou a } \\
\text { motivação do paciente podem ser } \\
\text { riscos modificáveis para a peritonite. }\end{array}$ & $\begin{array}{l}\text { A higiene domiciliar, a técnica de troca e a motivação estavam } \\
\text { acima da média em } 53 \%, 56 \% \text { e } 60 \% \text {, respectivamente. } \\
\text { Pacientes deprimidos desmotivados foram significativamente } \\
\text { mais propensos a desenvolver peritonite em comparação com } \\
\text { pacientes deprimidos motivados. }\end{array}$ & $\begin{array}{l}\text { Falta de motivação previu peritonite particularmente se } \\
\text { associada à depressão. Mais estudos são necessários com foco } \\
\text { em esquemas específicos de pontuação de motivação e apoio } \\
\text { psicossocial que pode levar a melhores resultados. }\end{array}$ \\
\hline $\begin{array}{l}\text { The home visit in } \\
\text { peritoneal dialysis: } \\
\text { relevant aspects to } \\
\text { nursing care }\end{array}$ & $\begin{array}{l}\text { Descrever a visita domiciliar (VD) em } \\
\text { diálise peritoneal } \\
\text { (DP) a partir do olhar dos usuários } \\
\text { que ingressam em diálise peritoneal } \\
\text { ambulatorial e discutir o significado } \\
\text { da VD para tais sujeitos. }\end{array}$ & $\begin{array}{l}\text { Percebe-se a visita domiciliar como uma dimensão subjetiva } \\
\text { dos usuários que realizam diálise peritoneal e de seus } \\
\text { familiares. }\end{array}$ & $\begin{array}{l}\text { A Visita domiciliar é um momento rico de interação enfermeiro e } \\
\text { usuário e uma grande ferramenta para a otimização do cuidado } \\
\text { com o indivíduo em seu domicílio. }\end{array}$ \\
\hline $\begin{array}{l}\text { Evaluation of real-time } \\
\text { use of electronic } \\
\text { patient-reported } \\
\text { outcome data by nurses } \\
\text { with patients in home } \\
\text { dialysis clinics. }\end{array}$ & $\begin{array}{l}\text { Avaliar as perspectivas dos } \\
\text { enfermeiros sobre a utilidade e o } \\
\text { impacto da administração do ePRO } \\
\text { em clínicas de diálise domiciliar e } \\
\text { avaliar a percepção do paciente } \\
\text { sobre a satisfação com o cuidado de } \\
\text { enfermagem após o uso de ePROS }\end{array}$ & $\begin{array}{l}\text { Os cinco temas que emergiram das entrevistas com os } \\
\text { enfermeiros: melhorar o foco dos enfermeiros, direcionar o } \\
\text { acompanhamento interdisciplinar, oferecer apoio aos pacientes } \\
\text { durante o processo, interpretar os resultados da exibição visual e } \\
\text { integrar-se ao fluxo de trabalho. As pontuações no Questionário } \\
\text { do Cliente sugeriram que os pacientes acreditavam que recebiam } \\
\text { atendimento excelente (97\%) e que os enfermeiros entendiam } \\
\text { perfeitamente suas necessidades }(90,9 \%) \text {. }\end{array}$ & $\begin{array}{l}\text { Os enfermeiros relataram que o compartilhamento de dados do } \\
\text { e PRO em tempo real informava sua prática. Embora não tenha } \\
\text { havido alteração estatisticamente significativa, alguns pacientes } \\
\text { relataram mudanças e benefícios com o uso de ePROs. Mais } \\
\text { pesquisas são necessárias para fornecer orientação sobre como } \\
\text { os dados do ePRO podem melhorar os cuidados centrados na } \\
\text { pessoa. }\end{array}$ \\
\hline
\end{tabular}


ciente quanto do familiar, devido a isso é importante a execução de avaliações periódicas no domicílio, afim de evitar complicaçóes ao paciente. ${ }^{(19)}$

Pacientes deprimidos e desmotivados foram significativamente mais propensos a desenvolver peritonite em comparação com pacientes deprimidos motivados, assim a falta de motivação pode desenvolver peritonite se associada à depressão. ${ }^{(20)}$ Porém, mais estudos devem ser realizados com foco nesse aspecto psicossocial.

\section{Discussão}

A diálise peritoneal é uma terapia domiciliar e a sobrevida do paciente está relacionada à adesão, entendimento e aceitação do paciente ao tratamento e a indicação da diálise peritoneal no domicílio. A presença de um programa de VD pode melhorar a aderência ao tratamento e os seus resultados. ${ }^{(17)}$ Os acahados dos estudos mostram que a educação em saúde realizada por enfermeiros contrubui para o melhor conhecimento dos pacientes, familiares e cuidadores em diversas situaçóes de cuidado domiciliar. ${ }^{(7,16)} \mathrm{O}$ treinamento é um dos determinantes para o sucesso da terapia, sendo fundamental na prevenção da peritonite. ${ }^{(7)}$ Ao iniciar o tratamento na DP domiciliar, todo paciente passa por treinamento realizado pelo enfermeiro do centro de diálise. Este treinamento é seguido pelas recomendaçóes das diretrizes da International Society for Peritoneal Dialysis (ISPD), ${ }^{(21)}$ sendo constituído de aulas teóricas e práticas, adequadas de acordo com a necessidades de cada paciente e/ou familiar, para que haja a compreensão do procedimento. ${ }^{(22)} \mathrm{Um}$ estudo mostrou que a depressão pode desencadear a peritonite em pacientes em $\mathrm{DP} .{ }^{(20)} \mathrm{A}$ depressão pode levar aumento na mortalidade e morbidade nos pacientes em diálise, comprometendo a aderência ao tratamento. $^{(23)}$

Nesse sentido, a enfermagem precisa percorrer um caminho para focalizar seus problemas específicos, utilizando referenciais teóricos, de forma a possibilitar uma estrutura de conhecimentos que fundamente sua prática. A Teoria de Enfermagem de Dorothea Orem surgiu nos anos 50 e tem como conceito básico o autocuidado. Ela tem sido abordada em diversos estudos realizados com pacientes que apresentam o diagnóstico de DRC, pois possui conceitos acerca do autocuidado ou déficit de autocuidado. ${ }^{(24,25)}$ Os enfermeiros apropriam-se dos conceitos de Orem para o planejamento do cuidado e implementação das açôes de enfermagem necessárias ao atendimento da demanda terapêutica. A identificação dos déficits de autocuidado pelo enfermeiro corrobora para o apoio e capacitação dos familiares como agentes do autocuidado do paciente, quando os requisitos estiveram diminuídos ou ausentes. As taxas de peritonite foram citadas por autores, ${ }^{(17,20)}$ mas somente $\mathrm{um}^{(18)}$ avaliou o efeito de um programa de visita domiciliar na técnica de diálise peritoneal, no efeito da adesão e os insucessos. A Portaria no 385, de 25 de abril de 2016, redefine a $\mathrm{AD}$ ao paciente em $\mathrm{DP}$ no âmbito do SUS, na modalidade de AD. O Serviço de Atenção Domiciliar (SAD) é caracterizado pelo conjunto de açóes de promoção da saúde, prevenção e tratamento de doenças, além da reabilitação da saúde que é realizada em domicílio, visando à continuidade e à integralidade de cuidado. ${ }^{(26)}$

Um estudo identificou déficits de conhecimento no procedimento de dialise peritoneal, tanto do paciente quanto do familiar. ${ }^{(19)}$ Como prática institucional a VD contribui satisfatoriamente para: o seguimento do tratamento no domicílio; possibilitou conhecer e acompanhar a realidade das famílias; interviu o mais rápido possível em falhas detectadas; esclareceu dúvidas com enfoque educativo. A avaliação socioeconômica realizada na VD ofereceu suporte ao paciente e família com finalidade de cuidado específico. ${ }^{(3,27)} \mathrm{A}$ realização periódica da VD mantém o paciente e/ou cuidador capacitados e supervisionados pela equipe de enfermagem em relação às condições do domicílio, equipamentos, insumos (materiais de higiene e limpeza). A manutenção das condiçôes adequadas possibilita a realização efetiva do procedimento domiciliar de DP. No contexto, a VD é um momento rico de troca de saberes entre profissionais e paciente/cuidador que podem participar de forma ativa, crítica, questionadora e refletirem sobre as reais necessidades, como sujeitos transformadores 
do processo saúde. ${ }^{(3,28)}$ Recomenda-se a realização de relatos e de estudos primários dessa temática, a fim de demosntrar e contribuir para a identificação das melhores evidências científicas. Estudos primários sobre o tema investigado apresentou-se limitado na literatura nacional e internacional, o que indica a necessidade de implementtar esforços e estimular a divulgação de psquisas e resultados obtidos. A pergunta de pesquisa da revisão mostrou a necessidade da presença o enfermeiro como profisisonal da equipe de saúde e imprescindível no planejamento da VD como responsável pela avaliação dos cuidados a serem implementados. A síntese dos conhecimentos da presente revisão corrobora para que a VD realizada pelo enfermeiro torne-se ação efetiva e holística ao paciente e família, de forma a assegurar o autocuidado e a manutenção do tratamento proposto, assim como a diminuição da falta de adesão e de complicaçôes. Como limitaçóes, não foram identificados estudos que abordassem a visita domiciliar como suporte da assistência de enfermagem em diálise peritoneal.

\section{Conclusão}

Os estudos mostraram que a educação em saúde do paciente, familiares e cuidadores em diálise peritoneal domiciliar é essencial para a prevenção da peritonite. Para que isso ocorra de maneira efetiva, é necessário que o profissional de saúde, em especial o de enfermeiro, esteja capacitado para estes cuidados. $\mathrm{O}$ enfermeiro presta assistência de forma holística ao paciente e familiares e, assegura a manutenção do tratamento proposto. Intervir preventivamente por meio da educação continuada é fato imprescindível para o tratamento em domicílio. A presente revisão integrativa identificou estudos descritivos, quantitativos, qualitativos e de revisóes de literatura, com níveis de evidencia V, VI e VII. Destaca-se que o estado da arte do tema foi demonstrado, o que corrobora para estimular o desenvolvimento de futuros estudos com níveis de evidência I, II e III, além de fornecer subsídios para a compreensão e adesão dos pacientes ao tratamento e prescrição em diálise peritoneal domiciliar.

\section{Referências}

1. Brasil. Datasus. Manutenção e acompanhamento domiciliar de paciente submetido a dpa/dpac [Internet]. Brasilia (DF): Datasus; [citado 2015 Abr 1]. Disponível em: http://tabnet.datasus.gov.br/cgi/ tabcgi.exe?sia/cnv/qbuf.def

2. Abreu RC, Pereira ER, Gabriel DP, Caramori CA, Barretti P, Caramori JC. Influence of Training on the Progression of Peritoneal Dialysis. Braz $\mathrm{J}$ Nephrol. 2008;30(2):126-31.

3. Cunha LP, Silva FV, Santos FK, Pires AS, Leone DR, Silva LC. The home visit in peritoneal dialysis: relevant aspects to nursing care. Rev Fund Care Online. 2017;9(1):128-36.

4. Dimkovic N, Oreopoulos D. Management of elderly patients with endstage kidney disease. Semin Nephrol. 2009;29(6):643-9.

5. Wagner M, Ansell D, Kent DM, Griffith JL, Naimark D, Wanner C, et al. Predicting mortality in incident dialysis patients: an analysis of the United Kingdom Renal Registry. Am J Kidney Dis. 2011;57(6):894902.

6. Kofteridis DP, Valachis A, Perakis K, Maraki S, Daphnis E, Samonis G. Peritoneal dialysis-associated peritonitis: clinical features and predictors of outcome. Int J Infect Dis. 2010;25;14(6):e489-93.

7. Mendes KD, Silveira RC, Galvão CM. Revisão integrativa: método de pesquisa para a incorporação de evidências na saúde e na enfermagem. Texto Contexto Enferm. 2008;17(4):758-64.

8. Moher D, Liberati A, Tetzlaff J, Altman DG; PRISMA Group. Preferred reporting items for systematic reviews and meta-analyses: the PRISMA statement. Int J Surg. 2010;8(5):336-41.

9. Malta M, Cardoso LO, Bastos FI, Magnanini MM, Silva CM. STROBE initiative: guidelines on reporting observational studies. Rev Saude Publica. 2010;44(3):559-65.

10. Tong A, Sainsbury $P$, Craig J. Consolidated criteria for reporting qualitative research (COREQ): a 32-item checklist for interviews and focus groups. Int J Qual Health Care. 2007;19(6):349-57.

11. Melnyk BM, Fineout-Overholt E. Evidence-based practice in nursing \& healthcare: a guide to best practice. Philadelphia: Lippincot Williams \& Wilkins; 2005. Making the case for evidence-based practice; p. 3-24.

12. Andrade AM, Silva KL, Seixas CT, Braga PP. Nursing practice in home care: an integrative literature review. Rev Bras Enferm. 2017;70(1):210-9.

13. Araújo FE, Neto VL, Mendonça AE, Lins TL, Granjeiro FM. As práticas assistências de enfermagem na diálise peritoneal: uma revisão. Rev Enferm UFPI. 2015;4(1):111-6.

14. François K, Bargman JM. Evaluating the benefits of home-based peritoneal dialysis. Int J Nephrol Renovasc Dis. 2014;7:447-55.

15. Schick-Makaroff K, Molzahn AE. Evaluation of real-time use of electronic patient-reported outcome data by nurses with patients in home dialysis clinics. BMC Health Serv Res. 2017;17(1):439.

16. Varela JU, Vidal MR, Pedreros MC. Perfil biosociodemografico y valoracion de necesidades en pacientes em dialisis peritoneal. Cienc Enferm. 2014;20(1):89-95.

17. Montilla LA, Carrillo NL, Jiménez DM, Jiménez LM, Zapata MA, Restrepo JM. Safety automated peritoneal dialysis-DPA in children by primary caregivers home. Enfermería Global. 2014;34(Abr):104-13.

18. Martino F, Adibelli Z, Mason G, Nayak A, Ariyanon W, Rettore E, et al. Home visit program improves technique survival in peritoneal dialysis. Blood Purif. 2014;37(4):286-90. 
19. Meza WJ, Cervantes AL, Reyna MA, Salinas MM. Conocimiento teórico y apego al procedimiento de diálisis peritoneal del paciente 0 su familiar. Enferm Nefrol. 2015;18(2):130-6.

20. Paudel K, Namagondlu G, Samad N, McKitty K, Fan SL. Lack of motivation: a new modifiable risk factor for peritonitis in patients undergoing peritoneal dialysis? J Ren Care. 2015;41(1):33-42.

21. Bernardini J, Price V, Figueiredo A; International Society for Peritoneal Dialysis (ISPD) Nursing Liaison Committee. Peritoneal dialysis patient training, 2006. Perit Dial Int. 2006;26(6):625-32.

22. Figueiredo AE, Moraes TP, Bernardini J, Poli-de-Figueiredo CE, Barretti $\mathrm{P}$, Olandoski M, et al.; BRAZPD Investigators. Impact of patient training patterns on peritonitis rates in a large national cohort study. Nephrol Dial Transplant. 2015;30(1):137-42.

23. Stasiak CE, Bazan KS, Kuss RS, Schuinski AF, Baroni G. Prevalence of anxiety and depression and its comorbidities in patients with chronic kidney disease on hemodialysis and peritoneal dialysis. J Bras Nefrol. 2014;36(3):325-31.
24. Figueiredo $A E$, Kroth L, Lopes MH. Diálise peritoneal: educação do paciente baseada na teoria do autocuidado. Sci Med (Porto Alegre). 2005;15(3):198-202.

25. Aggleton P, Chalmers $H$. Orem's self-care model of nursing. In: Aggleton P, Chalmers $\mathrm{H}$, editors. Nursing models and the nursing process. London: MacMillan Education; 1990. p. 59-70.

26. Brasil. Ministério da Saúde. Portaria n 385, de 25 de abril de 2016. Redefine a Atenção Domiciliar no âmbito do Sistema Único de Saúde e atualiza as equipes habilitadas. Brasília (DF): Ministério da Saúde; 2016.

27. Sadala ML, Bruzos GA, Pereira ER, Bucuvic EM. A experiência vivida pelos pacientes em diálise peritoneal domiciliar: uma abordagem fenomenológica. Rev Lat Am Enfermagem. 2012;20(1):68-75.

28. Santos FK, Glaucia Valente Valadares GV. Getting to know the world of those coping with peritoneal dialysis: symbolic links in their everyday life. Rev Enferm UERJ. 2011;19(3):473-8. 